\title{
Remarks on soil structure in relation to zero-tillage
}

\author{
F. R. Boone and H. Kuipers \\ Tillage Laboratory, Department of Agricultural Engineering, Agricultural University, \\ Wageningen, the Netherlands
}

Received: 21 October 1970

\section{Summary}

Soil tillage is influencing mean pore space and homogeneity of soil structure. Space requirements of roots are very small and therefore only a minor part of the soil needs to be in a favourable state for root development, if only this part is adequately distributed throughout the whole soil mass.

It is suggested that soil tillage normally creates a sufficiently heterogeneous soil structure that root development is not hampered. If the main tillage operation is left behind, soil structure will gradually become more homogeneous. Research should aim at defining critical situations in terms of homogeneity rather than in mean pore space. In future it will be necessary to give a well founded advise on soil tillage, because herbicides will offer an alternative as far as weed control is concerned.

\section{Introduction}

There are at least two reasons for an increasing importance of the knowledge about the influence of soil tillage on plant growth. In the first place there is a growing need for a sound motivation (Kuipers, 1963). If soil tillage raises soil fertility it should be known which processes play a role and to what extend and whether soil tillage is the most economical way to reach this higher productivity.

In the second place there is a growing possibility to change soil structure (Czeratzki, 1969). Herbicides will make soil tillage more dispensable as a method of weed control and therefore the amount of tillage is likely to diminish. This may increase soil density. On the other hand new developments in farm machinery will make it possible to reduce the number of trips over the field considerably. This will result in less compaction. Therefore the range of densities that can be obtained in practice will increase. The present equilibrium between the loosening effect of tillage operations and the compaction effect of the traffic in the field seems to be primarily determined by traffic involved in seedbed preparation in spring (Andersson et al., 1966; Kuipers et al., 1963). However a change as well in the degree of compaction as in the degree of soil loosening is likely to change the level of equilibrium. It should be possible to predict this change; at least we should have the means to describe the new situation quantitatively and we should know whether this change has any importance or not.

Data available up to now indicate that there is a range of soil densities that is suitable for high production (e.g. Boekel, 1963). Once we have brought our soils within that range it will be tried to treat the soil in such a way that we stay as long as possible within this range. Therefore we should know which situations are critical and which risks are involved for different crops, different soils and different climatic conditions, if a certain tillage operation is carried out or left behind. 
On soils with favourable physical properties, perhaps a general indication about the treatment of the soil for a certain crop sequence will be sufficient. On soils with a less stable soil structure it may be necessary to determine certain characteristics of the soil, before an advise can be given. Up to now few data are available that might serve as a base for a realistic soil tillage advise. It is not to be expected that results of a correlative nature, that may be obtained from experimental fields, will be sufficiently adequate, because of the large number of variables involved. It will therefore be necessary to understand the causative relations at least to a certain level. In the following it is tried to formulate a few ideas in this field from the present state of knowledge.

\section{The influence of tillage on density and structural homogeneity of the soil}

There are at least three different aspects in the effect of a main tillage operation: 1. loosening; 2 . crumbling or breaking; 3 . mixing. The implement, the way in which it is used and soil conditions determine each of these aspects. From height measurements and core samplings it appears that the loosening effect of the main tillage operation for the arable layer as a whole is considerable, but variability of pore space in one field is effected by crumbling, breaking and mixing and after the tillage operation variability appears to be very large (Kuipers et al., 1966).

If after a wet harvest a badly damaged sugar-beet field on a heavy soil is ploughed, the main effect seems to be that the soil is much more homogeneous after the operation, because puddles and deep tracks have disappeared and more or less regular furrows cover the field. However more in detail soil structure is indeed very heterogeneous. The smeared and puddled surface material, or after potato harvest the fine sieved soil material, is distributed more or less regularly through the arable layer, or it may form a dangerous layer in the bottom part. Big dense clods may alternate with large holes, filled or not with more or less crumbled soil.

On coarse textured-soils that crumble very well on ploughing soil structure is likely to be more homogeneous after ploughing.

The loosening of the arable layer as a whole diminishes rapidly in the first days after ploughing, but normally a considerable effect is still present in spring (Kuipers et al., 1963). It may be expected that soil-structural homogeneity will also be influenced by this settling process and also by freezing and thawing or eventually wetting and drying. However the relatively high pore space in early spring makes it very likely that homogeneity especially on a heavy soil is still rather low. In spring on seedbed preparation total pore space of the soil beneath the seedbed is reduced strongly by the traffic in the field, and this will also influence soil structural homogeneity. During the first trip the soil is compacted according to a certain pattern, leaving loose soil between the ruts. After the final trip in spring the soil surface is normally covered at least once by the tracks made and therefore pore space is reduced in the whole field. For the heavy clay soil the process will be that the large holes are filled up more or less by clods that may be broken by the pressures exerted by the wheels. Although it is not likely that these holes will be filled up to the same density as the rest of the soil, soil structural homogeneity will be increased by this process. On a light soil, that crumbled very well on ploughing, the first soil-compacting trip in spring may have decreased soil-structural homogeneity by introducing compacted strips, but here the final effect of spring operations on homogeneity is less clear.

Field observations of soil structure during the growing season reveal that sometimes the pattern of the plough furrows is clearly recognized, indicating that the effect of 
the plough on soil homogeneity is still present. If tillage operations are not carried out in the next years, pore space may be still further reduced and soil homogeneity may increase, but changes will be smaller each year and another equilibrium will be reached.

The question arises whether a critical condition is likely to be reached and if this critical condition will be sufficiently characterized by a critical density or that homogeneity must be taken into account.

\section{Critical density and homogeneity of the soil}

In research extreme conditions are often used to make relations more readily understood. If, however, a critical density of the soil, where the crop is a failure, is found from pot or plot trials, it is not sure that the same density in the field will give a comparable reaction, because under practical conditions the situation will generally be more complex. Failures on headlands and certain results on zero-tillage fields indicate that also under practical conditions, a critical density does exist.

In theory there is no critical density, because plants can even be grown on a medium that cannot be penetrated by roots, if only roots are protected for light and too high temperatures, if they are kept moist enough and well aerated, if nutrient supply is sufficient and if of course the plants are kept upright by some special device. The lower the level of the technical provisions, the more dependent on the possibilities for root development the plant will be.

Because during the growing period soil structure cannot be changed materially by mechanical means, soil structure should be such that even under unfavourable weather conditions there is a minimum risk that plant development will be hampered. It is clear, on the other hand, that only a relatively small part of the soil will have to be in a favourable condition. What part we will need may be deduced from the knowledge of plant requirements.

\section{Required part of the soil suited for root growth}

Plant roots should not only be able to penetrate the soil, growth should also be hampered as little as possible. In regions where the drying front moves fast into the soil the speed of development is of special importance. Important questions are: what resistance will be encountered by plant roots, is this resistance always high in a dense soil and what force can be exerted by plant roots?

In a homogeneous medium the maximum force exerted by plant roots under favourable aeration conditions is not very high. Root growth pressure, defined as root growth force per unit root cross-sectional area, averaged 9.4, 13.0 and 11.5 bars for cotton, peas and peanuts, respectively (Taylor et al., 1969). There are considerable differences between different plants of one variety and therefore these figures have the statistical nature of an average. If we measure the resistance in the top soil with a penetrometer a critical value for crop growth seems to be 20 or $30 \mathrm{~atm}$ (Taylor et al., 1964, 1966). The cones used are large as compared to the size of plant roots, and they are moved into the soil at relatively high speeds. Moreover they are not flexible and therefore they are a better indication for the difficulties a growing root may encounter than for the possibilities for a growing root to avoid these difficulties as they will try to do. Growing roots will follow pores and channels of a suitable size and only try to press the soil aside if there is no other possibility. Pores and channels suitable 
for root growth certainly need not be larger than about $1 \mathrm{~mm}$ and for a sufficiently large channel system only a small part of the soil is needed. $1 \%(\mathrm{v} / \mathrm{v})$ of the soil in a $20 \mathrm{~cm}$ deep arable layer is $20 \mathrm{~m}^{3} / \mathrm{ha}$, which would make about 20 tons of roots per ha! Therefore not more than $1 \%$ of the soil will be needed for a well-developed root system. This implies that a measurement of a highly variable soil parameter on a certain spot is not very likely to be really relevant for the root, and the average of a large number of such measurements will also not be suited for an adequate characterization, because the average eliminates the influence of extreme values, that in this case are likely to determine the relevant characteric. Nevertheless it is possible that under certain conditions there is a rather close relation between, for instance, mean total pore space and root development if soil-structural homogeneity is the same for the different objects. But a different distribution of the same amount of soil in a certain volume will not change mean total pore space, but mechanical resistance for root growth may be entirely different after the redistribution.

Another important point is that the part of the soil that is well suited for root development, must be continuous and distributed in such a way that it fits the root system. A system of channels starting at $10 \mathrm{~cm}$ depth is of no use and a system that is restricted to the top $10 \mathrm{~cm}$ of the soil may give a favourable carly development of the crop, but especially when water and nutrient supply of the crop is marginal, great risks are involved. Generally it is clear that it is dangerous when that part of the soil that cannot be penetrated by roots, is present in layers, especially if these layers are close to the surface. Although shrinkage cracks may appear more readily near the surface damage is very likely.

It is not sufficient that roots penetrate the soil. Roots should also be able to function properly. Therefore aeration is necessary. If we want to calculate what part of the soil we need for a sufficient aeration, we need to know how much oxygen is used and how much carbon oxide is produced by the soil and the crop and which concentrations can be tolerated.

For a long time it was thought that $\mathrm{CO}_{2}$ content should be lower than $1 \%(\mathrm{v} / \mathrm{v})$, but it was proved that for most plants, at any rate for a short time, concentrations up to $10 \%$ are not harmful. Even a small stimulating effect of concentrations of $1-3 \%(\mathrm{v} / \mathrm{v})$ was reported (e.g. Geisler, 1969). Higher concentrations will not often be found in practice, because $\mathrm{CO}_{2}$ is very soluble in water, contrary to $\mathrm{O}_{2}$. Therefore the $\mathrm{O}_{2}$ concentrations will drop to very low levels before a higher $\mathrm{CO}_{2}$ concentration than e.g. $3 \%$ is reached. $\mathrm{O}_{2}$ concentrations may even drop to low values without a decrease of dry-matter production. In lysimeters no decrease of $\mathrm{O}_{2}$ consumption, nor a growth depression was observed when the soil was sealed from the free air and $\mathrm{CO}_{2}$ was removed till nearly all $\mathrm{O}_{2}$ had been used (Brown et al., 1965). Although differences between plants have been reported and although there are differences in results for different conditions, it is likely that under normal conditions even a few $\%$ of $\mathrm{O}_{2}$ are already sufficient.

$\mathrm{O}_{2}$ consumption and $\mathrm{CO}_{2}$ production are very variable. During the growing season the soil uses $1.8-11 \mathrm{l} \mathrm{O}_{2}$. day-1. $\mathrm{m}^{-2}$ and the plants $2.8-5.61 \mathrm{O}_{2}$. day ${ }^{-1} \cdot \mathrm{m}^{-2}$ (Brown et al., 1965). A calculation of the total air volume required for diffusion will give a value of about $1.3 \%(\mathrm{v} / \mathrm{v})$, if under a homogeneous active layer of $25 \mathrm{~cm}$ soil with a production of $101 \mathrm{CO}_{2}$. day-1. $\mathrm{m}^{-2}$ (which is a high value for arable land). $\mathrm{CO}_{2}$ content is to be kept under $10 \%$ and solubility is neglected. For a critical value of $5 \% \mathrm{CO}_{2}$ $2.5 \%(\mathrm{v} / \mathrm{v})$ of air will be required, for $2 \% \mathrm{CO}_{2} 6.3 \%$ of air, and for $1 \%$ of $\mathrm{CO}_{2}$ $12.8 \%$ of air. From this it can be concluded that only a very small part of the 
arable layer is required for diffusion. This could be the same part of the soil as that suitable for penetration of the roots. At any rate these two parts should be in close contact.

These considerations therefore support the idea that only a rather small part of the arable layer needs to be suited for root growth, if only certain requirements of continuity and distribution are met.

A few remarks should be made:

In diffusion calculations it is assumed that there is a free connection between soil air and atmospheric air. However in soils diffusion drops to zero before air content is approaching this value, indicating that a certain part of the air is not contributing for diffusion, air is entrapped on wetting. According to information available as much as $10 \%(\mathrm{v} / \mathrm{v})$ of air can sometimes be entrapped (van Duin, 1956). Homogeneous surface layers of slaked or puddled soil will offer a high resistance for diffusion (Bakker et al., 1970). If these layers are thin and are not continuous over a considerable surface, their influence is restricted. As long as the volume of inactive air is small or is concentrated in certain spots in the soil, diffusion calculations are likely to be applicable.

It is supposed that there is a direct contact between air in the soil and plant roots. But we have to be aware of the fact that even a thin water-film around the root will give a considerable gradiant in $\mathrm{O}_{2}$ concentration, because diffusion in water is about $10^{4}$ times smaller than in air. Therefore even very high air contents still may be insufficient at very low water suctions (Grable et al., 1968). A light increase in water suction will diminish the size of the water-film considerably. Reality is complicated by the fact that there are also soil particles around the root, but nevertheless we can state that amelioration of soil structure by tillage will only make sense if water management of the soil is reasonable. This implies that soil-water suction should not be too low and at any rate there should be no water on the surface too long a time.

It is possible to calculate from rainfall data the probability of a certain surplus of water during a certain period for a soil with a given infiltration capacity (van Duin, 1956). For the Netherlands a $2-\mathrm{cm}$ surplus should be expected once in two years, if the infiltration capacity is as low as $1 \mathrm{~mm} / \mathrm{h}$ and it will take $40 \mathrm{~h}$ before this surplus has disappeared from the surface of the soil. Once in 10 years, the surplus will be $3 \mathrm{~cm}$ and there will be water on the surface for about 3 days.

If the soil has a mean air content of $5 \%(\mathrm{v} / \mathrm{v})$ over a depth of $1 \mathrm{~m}$, there will be about $10 \mathrm{I} \mathrm{O} / \mathrm{m}^{2}$. If $5 \mathrm{l} / \mathrm{m}^{2}$ is used per day, this will only be sufficient for 2 days. If drainage is sufficient, these low air contents will be present only in spring and then $\mathrm{O}_{2}$ consumption is low because of low temperatures and small root systems. Generally speaking in temporate regions the amount of air in the soil will be sufficient for 1-10 days on arable land.

In glasshouse culture where high amounts of sometimes readily decomposing organic material are mixed through the soil, where temperatures are high and root systems develope very rapidly, the amount of air in the soil might be exhausted in hours instead of in days, which stresses the necessity of a good aeration under these conditions.

An infiltration capacity of $1 \mathrm{~mm} / \mathrm{h}$ is of the same order as the capacity of one single hole of $1 \mathrm{~mm}^{2}$ per $\mathrm{m}^{2}$ of soil surface.

This explains why it is so difficult to make soil impermeable by mere compaction. Of course the amount of water that has to penetrate into the soil may be increased 
several times even in a flat country by small depressions of the surface, but generally a kind of blocking action of the channels in the soil by slaked or puddled soil material will be necessary to reduce the infiltration capacity on a certain spot sufficiently to account for puddles on the surface that last for a longer time. It is therefore readily understood that these more or less consistent surface puddles are likely to have a restricted size.

For aeration water on the surface is even more dangerous than water standing on a deeper layer that will leave a part of the root system in an aerated soil. It should be kept in mind that even very thin puddled layers may seal a surface completely if they are continuous. For an adequate drainage of the soil, however, a neglectible part of the pore space is strictly required in theory.

\section{Types of soil structure well suited for plant growth}

In a homogeneous compact soil mass only a few small mainly vertical channels per $\mathrm{m}^{2}$ occupying no more than $1 \%(\mathrm{v} / \mathrm{v})$ of the soil, would be necessary for a sufficient drainage and for space requirements of 20 tons of roots per ha. To give these roots a suitable environment in order to function properly as a part of a living plant, a channel system in a mainly vertical direction, fitting the pattern of root growth, occupying a few $\%(\mathrm{v} / \mathrm{v})$ of the soil would be sufficient. In this channel system mechanical resistance for plant roots would be irrelevant. The compact soil mass would facilitate moisture movement towards the roots, because capillary conductivity in an unsaturated soil is higher in a dense soil than in a loose soil. Therefore in dry areas a thin loose top layer or a mulch may be useful to prevent water loss in early spring.

Such a compact soil could be composed of closely fitting sharp edged, dense aggregates leaving a system of small interstices in between up to 5 or $10 \%(\mathrm{v} / \mathrm{v})$ of the soil. Such systems would be comparable to that of an undisturbed subsoil in a heavy clay soil or perhaps to a well-frozen compact clay soil. We could also think about a channel system created by biological activities.

Trafficability of such soils would be very high even in early spring, which might offer an opportunity for early sowing. We should consider whether this type of soil structure is realistic or not. Can we start from soils in this structural condition and will that situation be consisted or is this type of structure likely to develope if we stop to till the soil?

If we start from a stable structure in an undisturbed soil, like we will find in grassland, we will have the best chance for success. If we would not drive on the soil there is no reason to expect any problems with soil structure. In a bed system this might be possible but under present conditions we will have to accept a certain amount of traffic in the whole field. Our compact soil with a mainly vertical channel system may stand considerable vertical forces, but tractor tires often exert also large horizontal forces and soil movements are reported in a considerable volume of soil (e.g. Wong, 1967). These soil movements will depend strongly on the water content of the soil. The size of the channels that we consider is of course extremely small in comparison to tractor wheels. Even a large channel of $1 \mathrm{~mm}^{2}$ can be closed by a hardly observable movement of the soil. Especially close to the tires soil movement is rather complicated and the chance to disturb channels will be large. However close to the soil surface there is also the best chance of a restoration of physical nature by wetting and drying or freezing.

If we would have to till our soil, because too many channels have been closed, our 
tillage operation would result in a random arrangement of clods and aggregates. During winter and in early spring stabilization processes of a biological character will be small and traffic involved in seedbed preparation, fertilizing and sowing will compact the soil, that will still have a rather high moisture content, into an adhering mass in which the aggregates and clods are more or less destroyed whereas the holes in between are filled out more or less completely. Nevertheless total pore space and variability of pore space will be higher than before tillage, but the character of the system of large pores or channels has been changed fundamentally.

There will no longer be a mainly vertical system, and continuity is more problematic. In that case it is clear that the total volume of channels should be greater than needed from the theoretical point of view. If we now leave behind further tillage it is possible that the looser parts in the soil will disappear gradually. Total pore space will not change very much because only a minor part of the soil is involved in the changes. This process is more or less identical with the development of plough pans and the gradual decrease of a favourable effect of sub-soiling in the years after the operation. Not much is known about the rate at which these processes will occur under different conditions and to what extend the different aspects are relevant for different soils. However there is a strong feeling that we will need soil-structure characteristics that will enable us to predict where tillage operations are necessary and where they can be left behind. Perhaps for certain soils and certain climatic conditions and a certain crop rotation soil-physical conditions will never become a limiting factor. We should be sure about that. Under many other conditions the soil will be less tolerant and we should be able to give the limits. Finally there are soils where soil-physical conditions will be a real bottleneck and we should be able to indicate how the problems can be solved in the best way. The methods now available for soil-structure evaluation seem to be not sufficiently to the point with regard to an advise on soil tillage. We indicated in this paper the direction where we are in search for more effective characteristics.

The present development especially brought about by the introduction of rew herbicides enabling us to apply zero-tillage practices, indicate that we will need all research capacity available to keep ahead of the growing demand for exact information about how much and what kind of tillage is really necessary.

\section{Conclusion}

It does not seem likely that all tillage will disappear, because the technical possibilities of tillage in solving many problems involved in growing crops are too sophisticated, but it seems also very clear that chemical weed control will make the main tillage operation more dispensable. This will give the farmer more freedom to make a choice and it will be the task of agricultural research to provide him with information that will make it possible for him not to choose but to take the right decision. We hope that these articles will contribute to inspire especially research workers in this complicated field of research.

\section{References}

Andersson, S. \& I. Hakansson, 1966. Strukturdynamiken i matjorden. Grundförbättring 19: 191-228.

Bakker, J. W. \& A. P. Hidding, 1970. The influence of soil structure and air content on gas diffusion in soils. Neth. J. agric. Sci. $18: 37-48$. 
Boekel, P., 1963. Soil structure and plant growth. Neth. J. agric. Sci. 11: 120-127.

Brown, N. J., E. R. Fountaine \& M. R. Holden, 1965. The oxygen requirement of crop roots and soils under near field conditions. J. agric. Sci. 64: 195-203.

Czeratzki, W., 1969. Grundlagen und Probleme der Bodenbearbeitung. Landtechnik 24: 558-561.

Duin, R. H. A. van, 1956. On the influence of tillage on conduction of heat, diffusion of air and infiltration of water in soil. Versl. Landbk. Onderz. 62.7, p. 47 (Dutch: Eng. summ.) and p. 69.

Geisler, G., 1969. Die Wirkung der Kohlensäurekonzentration im Bereich niedriger Sauerstoffkonzentrationen in der Bodenluft auf das Sprosz- und Wurzelwachstum von Kulturpflanzen. $Z$. Acker-PflBau 130: 1-15.

Grable, A. R. \& E. G. Siemer, 1968. Effects of bulk density, aggregate size and soil water suction on oxygen diffusion, redox potentials and elongation of corn roots. Proc. Soil Sci. Soc. Am. 32: $180-186$.

Kuipers, H., 1963. The objectives of soil tillage. Neth. J. agric. Sci. 11: 91-96.

Kuipers, H. \& C. van Ouwerkerk, 1963. Total pore-space estimations in freshly ploughed soil. Neth. J. agric. Sci. 11: 45-53.

Kuipers, H., C. van Ouwerkerk \& G. J. Poesse, 1966. The characterization of soil structure changes produced by tillage operation. Grundförbättring 19:59-75.

Taylor, H. M. \& E. Burnett, 1964. Influence of soil strength on the root growth habits of plants. Soil Sci. 98: 174-180.

Taylor, H. M., G. M. Roberson \& J. J. Parker, 1966. Soil strength-root penetration relations for medium to coarse-textured soil materials. Soil Sci. 102: 18-22.

Taylor, H. M. \& L. F. Ratliff, 1969. Root growth pressures of cotton, peas and peanuts. Agron. J. 61: 398-402.

Wong Jo-Yung, 1967. Behaviour of soil beneath rigid wheels. J. agric. Engng Res. 12: 257-269. 